\title{
A comparative study of tissue glue and vicryl suture for conjunctival and scleral closure in conventional 20-gauge vitrectomy
}

${ }^{1}$ Department of Vitreoretinal Surgery, Ankara Ulucanlar Eye Research Hospital, Ankara, Turkey

${ }^{2}$ Department of Ophthalmology, Ankara University Faculty of Medicine, Ankara, Turkey

${ }^{3}$ Department of Biostatistics, Hacettepe University Faculty of Medicine, Ankara, Turkey

Correspondence:

Y Ozdamar,

Department of Vitreoretinal Surgery,

Eye Research Hospital,

Fazilet mah,

Açıkel sok.

Ankara 6110,

Turkey

Tel: + 903123183523 ;

Fax: + 903123124827

E-mail: yasemin_oz@

yahoo.com

Received: 11 December 2007

Accepted in revised form:

17 July 2008

Published online: 5

September 2008

\section{Abstract}

Aim To describe the use of tissue glue to close scleral and conjunctival wounds, and to compare the clinical outcomes using tissue glue and vicryl suture for closing these areas in conventional 20-gauge (G) vitrectomy.

Methods Thirty eyes of 30 patients were included in this study. The indications for vitreoretinal surgery were diabetic vitreous haemorrhage with severe vitreoretinal traction in 10 eyes, retinal detachment and proliferative vitreoretinopathy in $\mathbf{1 4}$ eyes, and vitreous opacity in 6 eyes. Tissue glue (Tisseel, Baxter AG Industries, Vienna, Austria) was used to attach scleral and conjunctival wounds in $\mathbf{1 5}$ eyes and vicryl sutures in $\mathbf{1 5}$ eyes. The patients were allotted into two subgroups as tissue glue group (TG) and vicryl suture group (VG). The sclerotomy sites were evaluated with ultrasound biomicroscopy (UBM) postoperatively in TG. Follow-up period was 2 months. The groups were statistically compared for ocular signs and symptoms by Mann-Whitney $U$-test.

Results No scleral wound leakage and conjunctival reattachment were observed at the end of the surgical procedure and during the follow-up period. No adverse effects were seen in TG. Abnormal fibrous ingrowth was not detected at the sclerotomy sites by means of UBM in TG. Patient comfort was significantly higher in TG than VG $(P<0.05)$. Conclusions Tissue glue has no adverse effects on ocular tissue and can be used as a substitute for suture materials, and the use of tissue glue decreases patient symptoms during the postoperative period after 20-G vitrectomy.
C Batman'1, Y Ozdamar'1, S Mutevelli', K Sonmez'1, G Zilelioglu² and J Karakaya ${ }^{3}$

Tissue glue can enable to perform sutureless surgery in the conventional 20-G vitrectomy. Eye (2009) 23, 1382-1387; doi:10.1038/eye.2008.252; published online 5 September 2008

Keywords: conventional vitrectomy; sclerotomy site; tissue glue; vicryl suture

Introduction

Conventional 20-gauge (G) vitrectomy system still remains popular in vitreoretinal surgery (VRS). Most of the vitreoretinal surgeons use 20-G instruments predominantly because they are still more versatile for the full spectrum of VRS. The sclerotomies are created with a 20-G microvitreoretinal blade directed perpendicular to the sclera at the inferotemporal, superotemporal, and superonasal quadrants after the conjunctival peritomy. At the end of the procedure, scleral and conjunctival wounds require sutures in 20-G conventional vitrectomy. ${ }^{1}$ The use of sutures for closing these wounds is associated with drawbacks such as postoperative discomfort and suture-related complications, and the eye often remains inflamed for days to weeks. It seems reasonable to use tissue adhesives as an alternative to suturing for conjunctival and scleral closure, and thus postoperative comfort improves and suture-related problems can be avoided.

Tissue adhesives are effective in attaching opposing wound edges quickly. Biologic adhesives (eg, fibrin-based adhesives) have more significant advantages and are used to achieve haemostasis and to seal or glue tissues. Tisseel (Baxter AG Industries), fibrin sealant, 
has been proved to have haemostatic and sealing properties in many types of surgeries. ${ }^{2-5}$

In this study, we aimed to determine the efficacy of fibrin sealant (Tisseel) to close scleral and conjunctival wounds and reviewed our clinical experience with the use of fibrin sealant during VRS. In addition, we compared the results of fibrin sealant and vicryl suture for closure of these wounds in a $20-\mathrm{G}$ vitrectomy system.

\section{Materials and methods}

A consecutive series of 30 eyes undergoing 20-G conventional vitrectomy were included in this study. Detailed preoperative examinations including bestcorrected Snellen visual acuity, biomicroscopic anterior segment examination, dilated posterior segment examination, and intraocular pressure (IOP) measurements were performed. All surgical procedures were performed by the same surgeon (CB). The patients were allotted into two subgroups as tissue glue group (TG) and vicryl suture group (VG). Tissue glue was used to close conjunctival and scleral wounds in 15 eyes. In VG, scleral wounds were sutured by 6-0 vicryl sutures and conjunctival wounds by $8-0$ vicryl sutures in another 15 eyes.

\section{Preparation of fibrin tissue glue}

Tisseel kit is a commercially available kit, which consists of two vials containing solvents and two vials containing powders. The Tisseel powder, consisting of fibrinogen, plasminogen, plasma fibroectin, and factor XIII, is reconstituted with the aprotinin solvent, and thrombin powder is reconstituted with calcium chloride solution according to the manufacturer's instructions. Each solution is placed in one of the dublojet syringe systems. Their contents are mixed in appropriate concentrations in the application needle of this system. The dublojet system allows simultaneous injection of the two components. Tisseel fibrin sealant, commercially available, was prepared according to the manufacturer's instructions in this study.

\section{Surgical technique}

All patients were operated under retrobulbar anaesthesia with lidocaine. After $360^{\circ}$ conjunctival peritomy, the episcleral surface was cauterised to prepare a bed for non-bleeding sclerotomy and then, three sclerotomies were performed with a 20-G microvitreoretinotomy knife in the inferotemporal, superotemporal, and superonasal quadrants. Pars plana vitrectomy was performed with a $20-\mathrm{G}$ vitreous cutter. In this system, 20-G surgical instruments were used for VRS. Retinal attachment was achieved by using perfluorodecaline liquid. The epiretinal membrane and internal limiting membrane were removed and endolaser photocoagulation was performed when required. Perfluorodecaline-air-silicone oil exchange (5000 or $1000 \mathrm{cs}$ ) was performed in 15 eyes and perfluorodecaline-air- $\mathrm{C}_{3} \mathrm{~F}_{8}$ exchange in 10 eyes. The vitreous space was filled with a balanced salt solution (BSS) in five eyes. Scleral buckling was combined with vitrectomy in 10 eyes.

In TG, after each of the entry site cannulas was removed, one drop of fibrin glue was immediately applied over the edges of each sclerotomy; these edges were opposed gently with forceps for $45 \mathrm{~s}$ for firm adhesion in each sclerotomy (Figure 1a and b). Excess sealant was excised with scissors. The wound was checked for leakage at the sclerotomy sites. A sponge was used to apply gentle pressure to the edges of the scleral incision and no wound leakage was shown (Figure 1c). Then, three or four drops of fibrin glue were injected on the bare sclera near the limbus all over $360^{\circ}$ and the conjunctival edges were immediately attached to opposing edges and kept pressed for 5-10s (Figure 1d). Surgery was completed after the closure of conjunctival wounds. In suture group, sclerotomies were closed using 6-0 vicryl suture. After no wound leakage was shown, conjunctiva was closed using the 8-0 vicryl suture.

Postoperatively, a pressure patch was applied for $24 \mathrm{~h}$. Following the procedure, the patients received oral ciprofloxacin (250 mg twice daily) for 2 weeks, oral indomethacin (75 mg once daily) for 1 week, frequent instillations of topical prednisolone acetate $1 \%$ for 8 weeks, and topical fucidic acid twice daily for 1 month.

Postoperative follow-up examinations were performed $1,3,7,14,30$, and 60 days after surgery. Best-corrected visual acuity, IOPs, and slit-lamp biomicroscopy of anterior segment and fundus findings, and wound leakage at sclerotomy sites were recorded for each patient. All postoperative examinations were performed by the same person The sclerotomy sites were evaluated with ultrasound biomicroscopy (UBM) 2 and 4 weeks after the operation in TG. All UBM examinations were carried out by one investigator (GZ). All patients were evaluated with regard to ocular symptoms and signs such as foreign body sensation, epiphora, and conjunctival hyperaemia at 1, 2, 3, and 4 weeks after surgery. These findings were graded according to patient satisfaction using a scale of 0-3 (0: complete satisfaction, 1: moderate satisfaction, 2: low satisfaction, and 3: unsatisfied). The groups were statistically compared for ocular signs and symptoms. Statistical differences were evaluated with Mann-Whitney $U$-test. Statistical analyses were performed with the Statistical Package for the Social Sciences for Windows (SPSS, version 10.0, Inc., Chicago, IL, USA). $P<0.05$ was considered statistically 

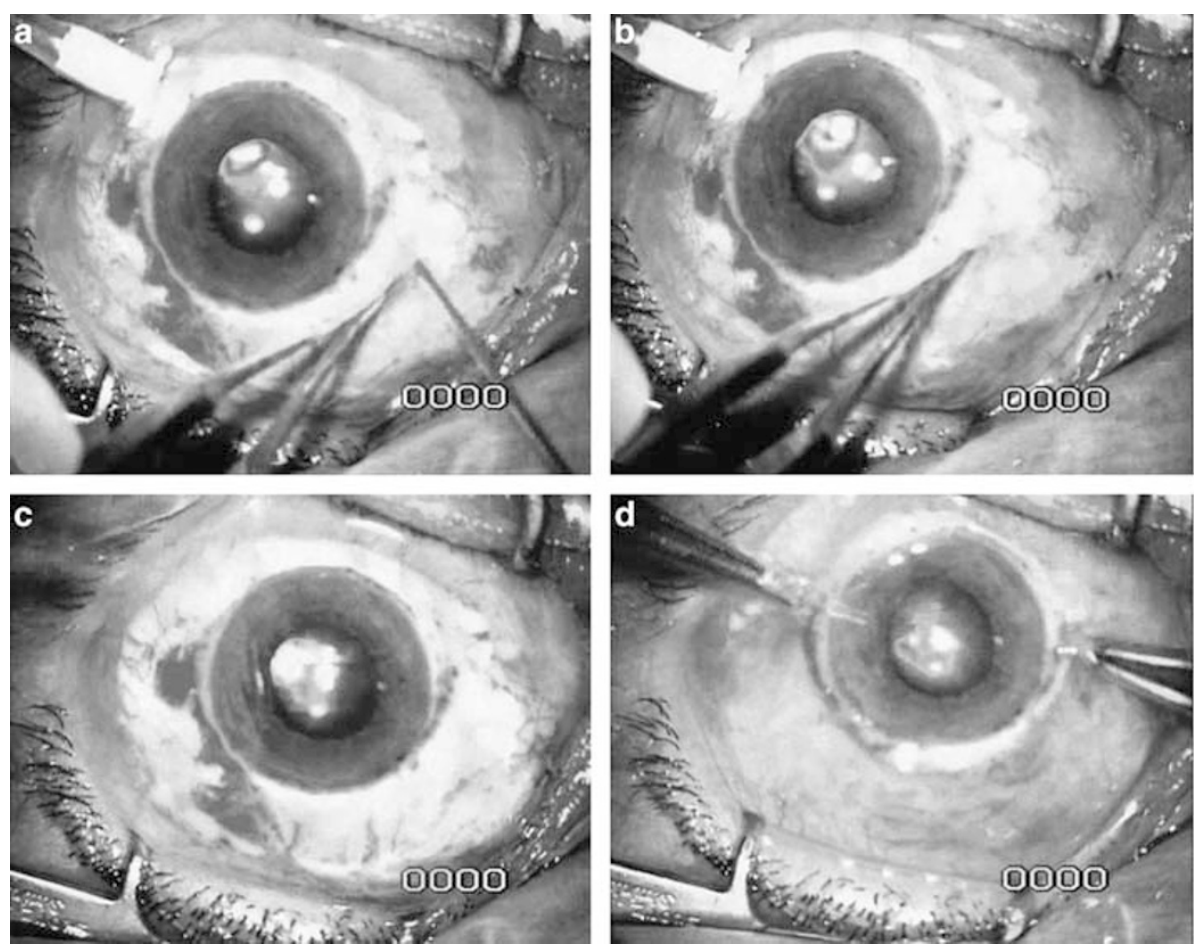

Figure 1 (a) Application of Tisseel over the edges of each sclerotomy in the last stage of 20-G vitrectomy. (b) Opposing sclerotomy edges gently with a forceps. (c) No leakage was shown at sclerotmy sites after closing with Tisseel. (d) The conjunctival edges were attached to opposing edges and kept pressed with a forceps.

significant. This study followed the tenets of the declaration of Helsinki and was approved by the institutional ethics committee. All patients gave written informed consents before the study.

\section{Results}

The indications for VRS were diabetic vitreous haemorrhage $(\mathrm{VH})$ with severe vitreoretinal traction in seven eyes, retinal detachment (RD) and proliferative vitreoretinopathy (PVR) in six eyes, and vitreous opacity in two eyes in TG. There was diabetic VH with severe vitreoretinal traction in three eyes, RD and PVR in eight eyes, and vitreous opacity in four eyes in VG. Of the 30 patients, $21(70 \%)$ were male and $9(30 \%)$ were female. The mean age of the patients was $58.7 \pm 7.3$ years (range 48-67 years). All patients were followed for 2 months.

No intraoperative complications occurred in any group. Silicone oil tamponade (5000 or $1000 \mathrm{cs}$ ) was used in 15 eyes, $20 \% \mathrm{C}_{3} \mathrm{~F}_{8}$ tamponade in 10 eyes, and BSS in 5 eyes. At the end of the surgical procedure, silicone oil, gas, and BSS leakage were not observed at sclerotomy sites in both the groups. None of the cases required sutures to close the conjunctival and scleral wounds in TG. In anterior segment examination, conjunctival hyperaemia was present in all eyes on the first

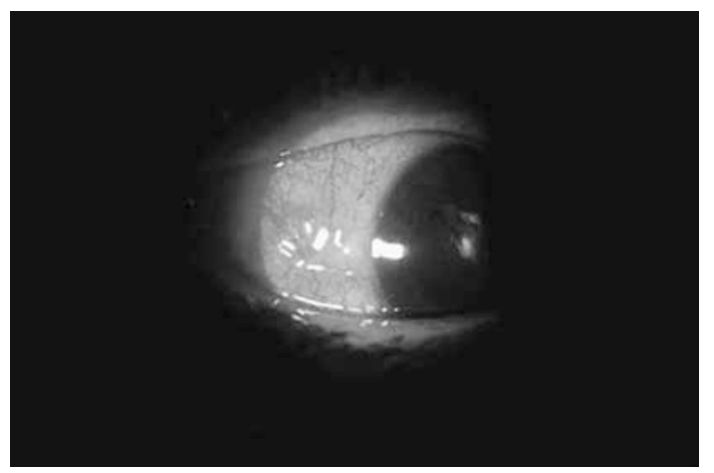

Figure 2 Postoperative slit-lamp view of the conjunctival edges attached by fibrin glue.

postoperative day. Conjunctival and scleral wounds were successfully attached and were intact in TG by the end of the follow-up period (Figure 2). Conjunctival bleb formation was not observed at the conjunctival surface on the sclerotomy sites in TG during and after surgery. Mild intraocular inflammation was present on the first postoperative day, which disappeared within the first week of the operations in both the groups. No hypotony was found in all eyes postoperatively. No side effects of tissue glue were encountered. 


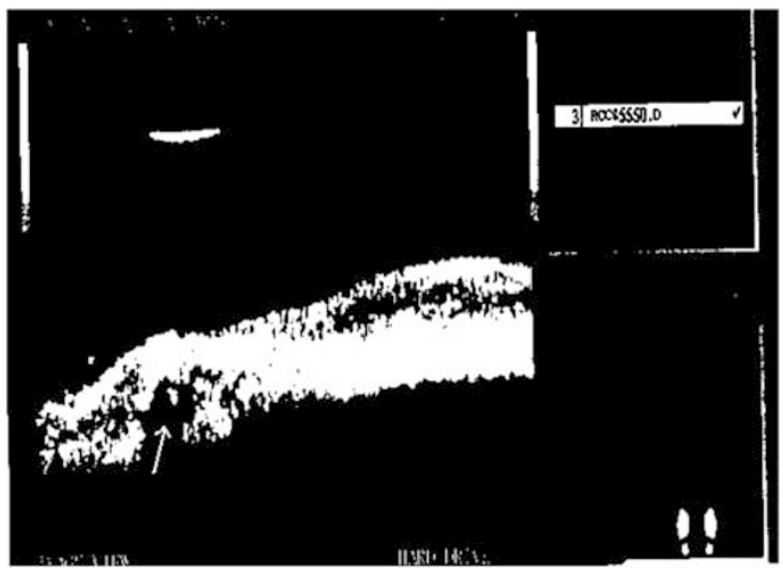

Figure 3 An ultrasound biomicroscopic image showing no abnormal fibrous proliferation at the sclerotomy site postoperatively (arrow: internal sclerotomy site).

Ultrasound biomicroscopy of scleral port sites showed no abnormal fibrous ingrowth in TG 2 and 4 weeks after $20-G$ vitrectomy (Figure 3). Ocular symptoms and signs were a lot milder in TG and were relieved more rapidly in TG than VG during the postoperative period. Ocular symptoms and signs were significantly lower $(P<0.05)$ in TG compared with VG.

\section{Discussion}

Suture and/or wound-related complications can be bothersome as they can lead to suture irritation, scleral necrosis, patient discomfort, granuloma formation, wound leakage, and postoperative astigmatism in VRS; therefore, the prevention of these problems is very important for vitreoretinal surgeons. Vitreoretinal surgical instruments and techniques have advanced dramatically to minimize these complications and sutureless vitrectomy systems such as conventional scleral tunnels, transconjunctival 20-, 23-, and 25-G vitrectomies have been used in VRS, recently. ${ }^{6-11}$ But, there are some drawbacks in each recent system. The conventionally reported sutureless vitrectomy by selfsealing 20-G sclerotomies uses the sclerotomy tunnel method; but, problems with scleral tunnels technique including wound leakage, extension, dehiscence, vitreous and/or retinal incarceration, haemorrhage, retinal tears, and dialysis have been reported and difficulty has been noted in passing the instruments through the sclerostomy tunnels. ${ }^{12}$ Transconjunctival sutureless vitrectomy systems such as 20, 23, and 25G are novel techniques in VRS but; transconjunctival 23and 25-G vitrectomies have been restricted because of limited capacity of surgical instruments, leading to a limitation of surgical indications. ${ }^{8,13,14}$
In transconjunctival $20-\mathrm{G}$ vitrectomies, conjunctival peritomy has not been performed but the use of suture is necessary to close conjunctival and scleral wounds. ${ }^{10,11}$ Also, transconjunctival sclerotomies may increase the risk of wound-related problems such as leakage, postoperative hypotony, and endophthalmitis. ${ }^{8,13-15}$ Today, conventional 20-G vitrectomy, surgical method with full and functional instrumentation is a gold standard especially for patients with complicated vitreoretinal disease requiring extensive intraocular tissue dissection and the combination of scleral buckle; but, this system requires closure of sclerotomy sites and conjunctival peritomy after the procedure. In this study, we thought the use of Tisseel as a fibrin sealant to close scleral and conjunctival wounds in patients requiring conventional 20-G vitrectomy and evaluated the tissuesealing property of fibrin sealant for closure of these wounds in conventional $20-\mathrm{G}$ vitrectomy.

Tissue adhesives can be subdivided into synthetic and biologic adhesives. Synthetic adhesives (cyanoacrylate derivatives) have mainly been used in the management of corneal perforation and severe thinning. Biologic adhesives (fibrin-based adhesives) have significant advantages. Fibrin sealant imitates the final stage of the normal physiological coagulation process and mimics physiological wound healing process. Fibrin sealant consist of two components, fibrinogen and thrombin solution. A clot is produced immediately upon contact of the two solutions. Fibrinogen is converted into fibrin monomers on a tissue surface by means of thrombin. Fibrin is then cross-linked by factor XIII to create a firm, stable fibrin network, independent of the patient's coagulation cascade. Aprotinin prevents rapid fibrinolysis. In the presence of calcium, thrombin catalyses the clotting of plasma coagulation proteins. The mechanical strength of a formed clot is thought to be related to the fibrinogen concentration in the fibrin sealant, which is 30 times that of normal plasma. In addition, the use of fibrin sealant might provide a critical balance of cellular regeneration and promote wound healing by acting as a biomatrix scaffold for cellular infiltrate, fibroblast proliferation and collagen deposition. Fibrin sealant induces no foreign body or toxic reaction. ${ }^{2-5,16,17}$

Tissue adhesives can successfully supplement or replace sutures and attach opposing wound edges quickly. ${ }^{2-5}$ Many studies on ophthalmic applications of tissue glue have been published. ${ }^{18-21}$ Literature review on fibrin sealant yielded a few reports on the use of the sealant for the closure of conjunctival openings in $20-\mathrm{G}$ vitrectomy. ${ }^{20,21}$ As far as we know, this is the first description of the use of fibrin sealant instead of sutures in the closure of sclerotomy sites in conventional 20-G vitrectomy. Vitreoretinal surgeons exert gentle pressure 
to the edges of the scleral incision to ensure the integrity of wound at the end of the vitrectomy and when no wound leakage is shown, sclerotomies are closed. However, scleral closure cannot be performed adequately despite the use of suture material, especially in patients with thin sclera and undergoing recurrent VRS, and scleral leakage may occur sometimes intraand/or postoperatively. The results of our study suggest that fibrin sealant can be effective in sealing in these patients.

In a review of published literature in ocular surgery, all studies concluded that fibrin sealant has a more comfortable postoperative period. None of the publications showed postoperative adverse or allergic reactions, bacterial infections, increased inflammation, or delayed healing. ${ }^{18-21}$ In our study, we observed that patient satisfaction with regard to ocular signs and symptoms, were significantly higher in TG than VG at all follow-up examinations and the patients with TG experienced a more comfortable postoperative period. We did not detect any allergic or adverse reactions in TG. In view of these studies, it is evident that tissue glue is superior to suture material in accelerating postoperative recovery.

Various histopathological studies have reported that Tisseel had no toxic effect on ocular tissue. ${ }^{22,23}$ There are some concerns regarding the safety of fibrin glue use, including theoretical risk of disease transmission and anaphylactic reaction. ${ }^{2-4}$ To the best our knowledge, no such cases have been documented. In this study, none of the patients had anaphylactic reaction or disease transmission during follow-up.

The peripheral retina, pars plana, and the vitreous base are difficult to visualize adequately by using indirect ophthalmoscopy and scleral indentation; but UBM provides excellent visualisation of these areas. ${ }^{24,25}$ Boker and Spitznas ${ }^{24}$ originally described the usage of UBM to examine the sclerotomy site after pars plana vitrectomy. In this study, abnormal fibrous ingrowth was not detected at sclerotomy sites in 20-G vitrectomy by means of UBM. Hence, according to UBM findings, we proposed that Tisseel causes no abnormal fibrous proliferation at sclerotomy sites.

In this study, we used Tisseel as an alternative to suture materials for the closure of conjunctival and scleral wounds in conventional $20-G$ vitrectomy. Scleral and conjunctival wounds were successfully closed and no wound leakage was observed. These sites were intact and none of the eyes had hypotony during the follow-up period. Also, we described a novel 20-G sutureless vitrectomy with the use of Tisseel. Favourable postoperative results were obtained by using Tisseel for the closure of these wounds. In conclusion, Tisseel decreases suture and/or wound closure-related complications and hastens postoperative recovery in VRS. Tisseel has no adverse effects on ocular tissue and might serve as an effective substitute in conjunctival and scleral wound closure in VRS. Use of tissue glue can enable ophthalmologists to perform sutureless surgery in the conventional $20-\mathrm{G}$ vitrectomy system.

\section{Acknowledgements}

The authors have no proprietary or financial interest in any of the products used in this study.

\section{References}

1 Charles S, Katz A, Wood B. Vitreous microsurgery. In: Charles S (ed). General Posterior Segment Techniques. Lippincott Williams and Wilkins: Philadelphia, 2002; pp 67-103.

2 Chan SM, Boisjoly H. Advances in the use of adhesives in ophthalmology. Curr Opin Ophthalmol 2004; 15: 305-310.

3 Radosevich M, Goubran HI, Burnouf T. Fibrin sealant: scientific rationale, production methods, properties, and current clinical use. Vox Sang 1997; 72: 133-143.

4 Clark RA. Fibrin and wound healing. Ann N Y Acad Sci 2001; 936: 355-367.

5 Jackson MR. Fibrin sealants in surgical practice: an overview. Am J Surg 2001; 182: 1S-7S.

6 Fujii GY, De Juan Jr E, Humayun MS, Pieramici DJ, Chang TS, Awh C et al. A new 25-gauge instrument system for transconjunctival sutureless vitrectomy surgery. Ophthalmology 2002; 109: 1807-1813.

7 Eckardt C. Transconjunctival sutureless 23-gauge vitrectomy. Retina 2005; 25: 208-211.

8 Hilton GF, Josephberg RG, Halperin LS, Madreperla SA, Brinton DA, Lee SS et al. Office-based sutureless transconjunctival pars plana vitrectomy. Retina 2002; 22: 725-732.

9 Chen JC. Sutureless pars plana vitrectomy through self-sealing sclerotomies. Arch Ophthalmol 1996; 114: 1273-1275.

10 Shimada H, Nakashizuka H, Nakajima M, Mori R, Mizutani Y. Twenty-gauge transconjunctival vitrectomy. Jpn J Ophthalmol 2005; 49: 257-260.

11 Gotzaridis EV. Three-port 20-gauge transconjunctival pars plana vitrectomy. Retina 2007; 27: 382-384.

12 Milibak T, Suveges I. Complications of sutureless pars plana vitrectomy through self-sealing sclerotomies. Arch Ophthalmol 1998; 116: 119.

13 O' Reilly P, Beatty S. Transconjunctival sutureless vitrectomy: initial experience and surgical tips. Eye 2007; 21: 518-521.

14 Amato JE, Akuman L. Incidence of complications in 25-gauge transconjunctival sutureless vitrectomy based on the surgical indications. Ophthalmic Surg Lasers Imaging 2007; 38: 100-102.

15 Taylor SR, Aylward GW. Endophthalmitis following 25-gauge vitrectomy. Eye 2005; 19: 1228-1229.

16 Busuttil RW. A comparison of antifibrinolytic agents used in hemostatic fibrin sealants. J Am Coll Surg 2003; 197: 1021-1028. 
17 Brown LF, Lanir N, McDonagh J, Tognazzi K, Dvorak AM, Dvorak HF. Fibroblast migration in fibrin gel matrices. Am J Pathol 1993; 142: 273-283.

18 Uy HS, Reyes JM, Flores JD, Lim-Bon-Siong R. Comparison of fibrin glue and sutures for attaching conjunctival autografts after pterygium excision. Ophthalmology 2005; 112: 667-671.

19 Mohan K, Malhi RK, Sharma A, Kumar S. Fibrin glue for conjunctival closure in strabismus surgery. J Pediatr Ophthalmol Strabismus 2003; 40: 158-160.

20 Mentens R, Stalmans P. Comparison of fibrin glue and sutures for conjunctival closure in pars plana vitrectomy. Am J Ophthalmol 2007; 144: 128-131.

21 Krzizok T. Fibrin glue for closing conjunctival wounds in ophthalmic surgery. Ophthalmologe 2004; 101: 1006-1010.
22 Bahar I, Weinberger D, Lusky M, Avisar R, Robinson A, Gaton D. Fibrin glue as a suture substitute: histological evaluation of trabeculectomy in rabbit eyes. Curr Eye Res 2006; 31: 31-36.

23 Kim MS, Kim JH. Effects of tissue adhesive (Tisseel) on corneal wound healing in lamellar keratoplasty in rabbits. Korean J Ophthalmol 1989; 3: 14-21.

24 Boker T, Spitznas M. Ultrasound biomicroscopy for examination of the sclerotomy site after pars plana vitrectomy. Am J Ophthalmol 1994; 118: 813-815.

25 Kwok AK, Tham CC, Loo AV, Fan DS, Lam DS. Ultrasound biomicroscopy of conventional and sutureless pars plana sclerotomies: a comparative and longitudinal study. Am J Ophthalmol 2001; 132: 172-177. 